
action lines to assist the clinician when the graph strays from the predetermined limits.

The section on the role of the cervix in labour is of specific interest to the authors and is one of the most informative sections of the book. The treatment of premature labour and operative obstetrics is well covered with many of the controversial aspects discussed in detail.

The authors have taken considerable care to deal with the controversial aspects of labour and to encourage readers to form their own opinions as this volume is intended for postgraduate readership.

The major omission of the book is maternal attitudes to care in labour. The authors make fleeting reference to some of the recent controversies about birth positions and mobility in labour. Consumer studies are alluded to but not really covered in detail.

In conclusion, however, I would recommend this as a good book; easy to read and one which will bring the postgraduate readership up to date with many of the controversies in obstetrics.

Y. B. GORDON,

The Garden Hospital, London NW4 IRX. obstetrics with graphical recording of the progress of labour and

Clinics in Oncology. Vol. 2, No. 3, November 1983. Transfusion Support Therapy

Edited by Charles A. Schiffer. Pp. xi +255 , illustrated. W. B. Saunders, London, 1983. £11.75.

This edition of Clinics in Oncology reviews the transfusion support treatment of intensively treated cancer patients. It deals with the problems of prolonged aplasia which are mainly encountered in patients undergoing remission, induction for acute leukaemia, or those being treated by autologous or allogeneic bone marrow transplantation. There are critical reviews of the use of platelets and granulocyte transfusion, an interesting review of antibiotic therapy and of coagulation abnormalities in cancer. Leucopheresis and cytophoresis are also the subject of separate chapters.

There is therefore much which will be of interest to the haematologist and oncologist. The chapters tend to be overreferenced: that on coagulation abnormalities is 30 pages long with 17 pages of references which makes the text exceedingly difficult to read. There is much sound advice however. I found the chapter on platelet transfusion in the alloimmunized patient very helpful, and those on granulocyte transfusion and antibiotic therapy to be well balanced and practical. I recommend this volume to all those involved in the intensive care of cancer patients and those who have to provide the investigations and support services.

R. L. Souhami, University College Hospital Medical School, London WCIE 6JJ.

Clinics in Immunology and Allergy. Vol. 3, No. 3, October 1983. Natural Mechanisms of Immunity

Edited by Ronald B. Herberman. Pp. vii +209 , illustrated. W. B. Saunders, London, 1983. $£ 11.75$.

The title of this book seems chosen to mystify. What, after all, are unnatural mechanisms of immunity? Immunologists, however, will know the editor's authority on the subject of natural killer (NK) cells. These are one of several mechanisms of 'natural immunity', defined by Dr Herberman as appearing 'spontaneously in most if not all individuals', not being 'dependent on known environmental stimuli', and showing 'considerable apparent specificity'. The natural immune system could be taken to include complement, interferon, NK cells and phagocytic cells, all of which can act independently of acquired $\mathrm{T}$ and $\mathrm{B}$ cell immunity to destroy or inhibit the growth of micro-organisms and cells.

Some of these mechanisms are succinctly and readably dealt with here. There are good chapters on the antibody-independent role of complement and the anti-microbial action of macrophages. Natural killer cells are given pride of place. These interesting lymphoid cells, distinguishable from $T$ and $B$ lymphocytes and monocytes, have lytic activity against some tumour, virus-transformed, and normal proliferating cells. Their biological role is uncertain, although attention has focussed on them as potential mediators of tumour surveillance. Diseases such as the $\mathrm{X}$-linked lymphoproliferative syndrome and immunosuppression-associated lymphomas begin to suggest their importance in man, at least in preventing uncontrolled lymphoproliferation. Other chapters seem less relevant to the book's purported theme. There are three on antibodies to tumours and oncogenic viruses. A summary of gastrointestinal protective mechanisms, though admirable, relates mostly to acquired immunity and the physiology of IgA induction and secretion.

There are good things here, for clinicians and immunopathologists; but there remains an unfilled place for a book drawing together what is known of mechanisms of natural immunity, which are active in the absence of acquired immunity (and probably have a longer evolutionary history), and which are of certain importance in defence against infection, in clearance of damaged tissue, and possibly in surveillance against neoplasia.

A. C. CAMPBell,

Leicester Royal Infirmary, Leicester LE1 5 WW.

\section{Modern Drug Treatment in Tuberculosis}

By J. D. Ross and N. W. Horne. 6th edn. Pp. 123. The Chest, Heart and Stroke Association. Tavistock House North, Tavistock Square, London WC1H 9JE, 1983. $£ 3.00$ (plus 50p postage and packing) (plus $£ 1.20$ surface mail oversees).

This book provides a modern authoritative and simple account of the chemotherapy of tuberculosis, a subject which has become increasingly complex as new drug regimens have been developed and the search for shorter and better chemotherapy regimens continues. There are now many programmes to suit the widely differing needs of both individual patients and of communities differing widely in economic, social and geographical factors. The authors cover this complex area with admirable clarity and brevity. The chemotherapy of many forms of tuberculosis including disease due to acquired drug resistance, the use of corticosteroids and drugs for the prophylaxis of tuberculosis are admirably reviewed by these wide and experienced clinicians.

The book provides much more than a guide to chemotherapy. In addition, there are excellent chapters on tuberculin testing, BCG vaccination and the control of tuberculosis. The slim volume will prove invaluable to anyone treating tuberculosis in both technically advanced and developing countries and will be helpful also to those concerned with tuberculosis prevention and control. It has the added advantage of being concise and cheap.

\section{K. M. Citron,}

Brompton Hospital,

London SW3 6HP.

\section{Palliative Care: The Management of Far-Advanced Illness}

Edited by Derek DoYle. Pp. vii + 536, illustrated. Croom Helm, London, 1984. $£ 25.00$.

There are now numerous books on terminal care. Most limit themselves to cancer. This book is exceptional, though not unique, in describing the care of patients with a wide variety of fatal conditions. It also extends the definition of terminal care to include those months, and sometimes years, when patients can be helped to live with dignity despite their disease. 